\title{
MODELOS DE ELECCIÓN: PROGRAMAS Y EXPERIENCIAS DE LIBERTAD DE ELECCIÓN EN ESTADOS UNIDOS
}

Resumen. Este artículo muestra, a través de experiencias educativas reales, la gran variedad de posibilidades que existen para llevar a la práctica un concepto tan amplio y ambiguo como la libertad de elección en educación. Otro de los intereses de estas páginas es presentar una clasificación de los modelos de programas de elección a partir de una pequeña representación de experiencias de cada tipo llevadas a cabo en Estados Unidos, el país que más ha impulsado los distintos sistemas de elección. El objetivo es ofrecer un instrumento útil para cualquiera que desee profundizar más en estos análisis, además de clarificar el campo terminológico que tanto dificulta la comprensión de esta temática. Esta sistematización puede ayudar a clarificar los heterogéneos modelos de elección y asociarlos con diferentes propósitos de los sistemas educativos.

Descriptotes: Política de la educación, libertad de elección, cheque escolar, Estados Unidos.

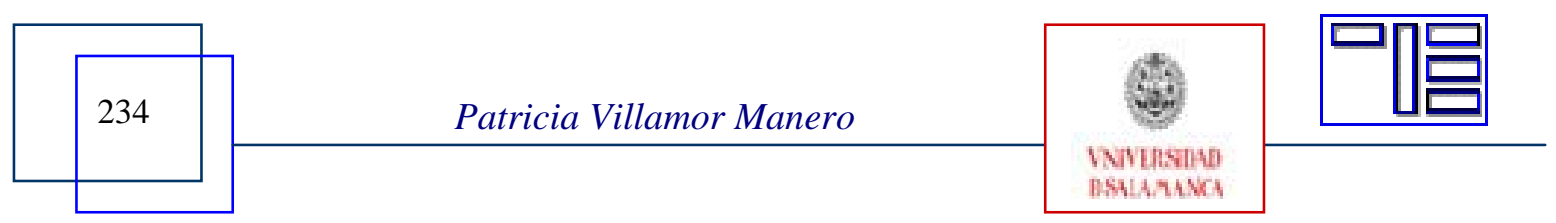




\section{MODEL OF CHOICE: PROGRAMS AND EXPERIENCES OF FREEDOM OF CHOICE IN THE UNITED STATES}

Abstract. This paper tries to show, through many examples, the great variety of possible forms to carry to the practice an ambiguous and so extensive concept as school choice in education. Other interest on this article is the classification of the school choice programs and the synthesis of each type of programs carried out in the United States, the country that harder has supported the different models of school choice. The objective is to offer an useful instrument for everybody who wishes to deepen more in these analyses, besides to clarify the terminological field what makes difficult the understanding of this issue. This systematization can help to clarify the heterogenous models of election and to associate each one with different intentions in the educational systems.

Keywords: Politics of education; school choice; vouchers; United States.

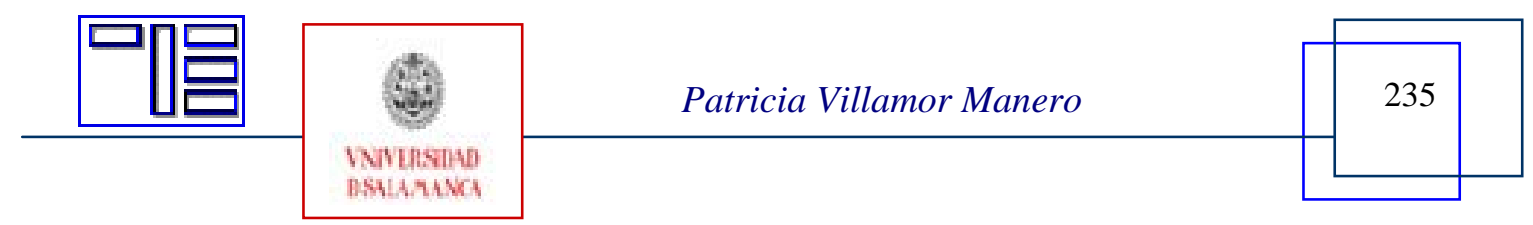




\section{MODÉLES DE CHOIX SCOLAIRE: PROGRAMMES ET EXPÉRIENCES DE LA LIBERTÉ DE CHOIX CHEZ ÉTATS-UNIS}

Cet article montre, par le biais des expériences pédagogiques, des possibilités qui existent pour mettre en oeuvre un concept si vaste et si ambigu que la liberté de choix en matière d'éducation. Un autre intérêt de ce site est de fournir un modèle de la classification des programmes de choix d'une petite représentation de chaque type d'expériences menées aux États-Unis, le pays qui a conduit les différents systèmes électoraux. L'objectif est de fournir un outil pour tous ceux qui veulent approfondir l'étude de cette analyse, en plus de préciser la terminologie domaine qui empêche la compréhension de cette question. Cette systématisation peut contribuer à clarifier l'hétérogénéité des modèles de choix et de s'associer avec des objectifs différents des systèmes éducatifs.

Mots clés: Politique de l'éducation, liberté de choix, chèques scolaire, USA.

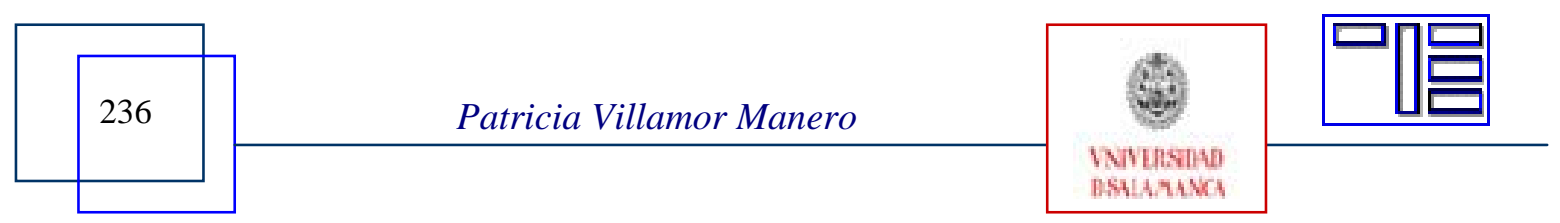




\title{
MODELOS DE ELECCIÓN: PROGRAMAS Y EXPERIENCIAS DE LIBERTAD DE ELECCIÓN EN ESTADOS UNIDOS
}

\author{
Patricia Villamar Manero \\ ptciavil@edu.ucm.es \\ Universidad Complutense de Madrid
}

\section{1.- INTRODUCCIÓN}

El concepto de school choice, traducido como elección de centro, ha sido definido de formas muy distintas por una gran cantidad de autores de todas las tendencias. Sin embargo, abarca mucho más que un sistema de bonos escolares (vouchers), con el que se le ha identificado desde que Milton Friedman ${ }^{1}$ lo hiciera por primera vez en los años sesenta, y puede concretarse en modelos con características diferentes adaptadas a los contextos donde se desarrolle. De hecho, aunque la expresión "school choice" parece referirse únicamente a las posibilidades de las familias para elegir uno u otro centro donde matricular a sus hijos, la libertad de elección en educación es un concepto más amplio que, además de abarcar el anterior, implica otras elecciones que pueden llevarse a cabo en educación, como la de la formación religiosa que se elige para los hijos, enseñanza en casa o en un centro autorizado, etc., aunque es evidente que, en la mayoría de estos casos, las elecciones realizadas significan también seleccionar un centro concreto. Además, desde el punto de vista pedagógico tiene muchas implicaciones como la necesaria diversidad de la oferta educativa para que las elecciones sean reales o la necesidad de autonomía de los centros para que puedan crear sus propios proyectos curriculares.

En la actualidad, el debate contemporáneo sobre sus beneficios y riesgos "está embarullado y desviado por una confusión básica respecto de qué implica exactamente school choice” (Henig, 1995, 175). De hecho, la elección significa cosas muy diferentes en cada situación y una de las dificultades al tratar con este asunto es aclarar la versión particular de libertad de elección que se está utilizando (Bennett, 1990, 126). Para realizar un análisis correcto de sus efectos, requisitos e implicaciones pedagógicas, además de las políticas educativas generales de cada país en relación con la libertad de elección de centros, es necesario conocer las experiencias y programas concretos que se han desarrollado en cada uno de ellos, pues como afirma Gaurin, en política se depende mucho de cómo los planes son puestos en práctica y de cómo los programas son incluidos en las instituciones particulares (Gaurin, 1999, 1).

Es difícil, sin embargo, encontrar bibliografía que aporte datos sobre muchas de estas experiencias. La revisión que presento en este artículo forma parte de una investigación más amplia sobre políticas y programas de elección en el contexto internacio$\mathrm{nal}^{2}$. Se incluyen en este espacio una clasificación propia de los modelos de programas de elección que se han experimentado y una pequeña representación de cada tipo de programas llevados a cabo en las escuelas. El objetivo es ofrecer un instrumento útil para cualquiera que desee profundizar más en estos análisis, además de clarificar el campo terminológico que tanto dificulta la comprensión de esta temática. Asimismo se pretende mostrar la variedad de caminos que hay para implantar los diferentes programas y los distintos objetivos a los que sirven. Esta variedad muestra el amplio conjunto

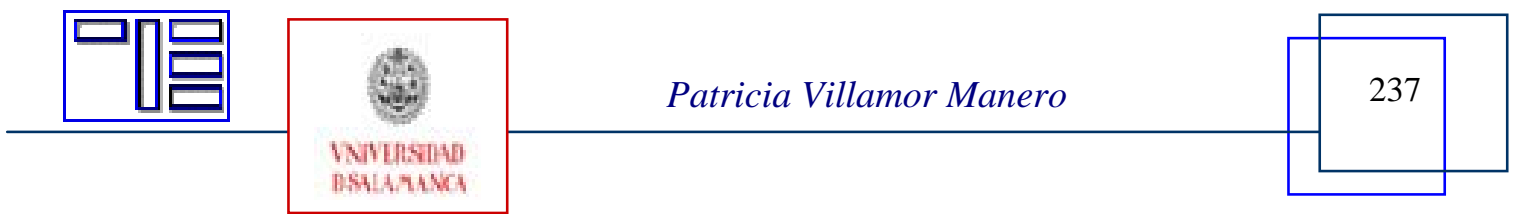


de concepciones educativas que se recogen bajo el concepto de la elección. Debido a la limitación espacial he tenido que seleccionar el número de experiencias incluidas en este análisis, limitándome a las más relevantes de Estados Unidos, país que mayor cantidad y diversidad de programas ha implementado.

La información sobre los programas está organizada en tablas para permitir una visión rápida de conjunto y evitar la confusión con las exhaustivas cifras y datos. Las tablas contienen información sobre el lugar y la fecha en que se desarrolló la iniciativa (si hay una fecha concreta de referencia), el nombre (si lo tiene), los objetivos fundamentales y una descripción muy breve de su funcionamiento o de las partes más destacadas del mismo, y por último, la categoría de modelos de elección en la que se inscribe. Se sigue un orden cronológico en la exposición, que ayuda a descubrir la evolución histórica del concepto y uso de la elección en los sistemas educativos. Aunque los objetivos y la descripción que se hace de los programas son muy diferentes entre sí, con la dificultad añadida de que en los documentos originales aparecen expresados con terminología distinta, he tratado de reducirlos a unos pocos para facilitar su comparación, encontrando las semejanzas y diferencias entre ellos. He unificado las expresiones "bono" y "beca”, al clasificar el tipo de programa al que se refieren. Aunque algunas experiencias son presentadas como becas de estudios, en realidad se definen con las mismas características y objetivos que los identificados como programas de bonos, ya que ambos subvencionan la enseñanza de un alumno en un centro público o privado. Como veremos, ambos se refieren al mismo tipo de diseño, al menos en lo que a extensión de la libertad de elección se refiere.

\section{2.- CATEGORÍAS DE PROGRAMAS INCLUIDAS EN LAS TABLAS}

Los programas de elección se pueden agrupar en cinco modelos:

- Modelos basados en la creación de escuelas con especialización curricular y/o organizativa.

Hay varios tipos de centros que habitualmente aparecen vinculados a estos modelos: las escuelas imán (magnet schools), las escuelas con contrato (charter schools), las escuelas temáticas, las escuelas alternativas y los centros que empiezan a denominarse especializados curricularmente. Sin embargo, podríamos considerar incluido cualquier programa encaminado a ampliar la diversidad de la oferta y la ampliación de la libertad de elección. Las escuelas imán surgieron en Estados Unidos durante los años 70 como mecanismo fundamental para combatir la segregación racial y mejorar la calidad de la educación (Raywid, 1989, 15). Su puesta en marcha pretendía que la elección sustituyera a los movimientos migratorios obligatorios sufridos por muchos estudiantes de color durante años, para que los centros admitieran una proporción equilibrada de cada raza representada en la población general (conocido como fenómeno del busing), aunque las escuelas desde las que provenían sufrían a veces un desequilibrio mayor al que se pretendía corregir. Al construir escuelas atractivas en barrios mayoritariamente negros, se intentó atraer a la población blanca para que matriculara voluntariamente a sus hijos en estos centros y conseguir un alumnado interacial. Las escuelas con contrato han sido denominadas así porque, para poder funcionar, deben establecer un contrato con el distrito escolar o con el gobierno del estado correspondiente. Su nombre original de charter schools, ha sido traducido como escuelas con contrato (Pini y Anderson, 1999),

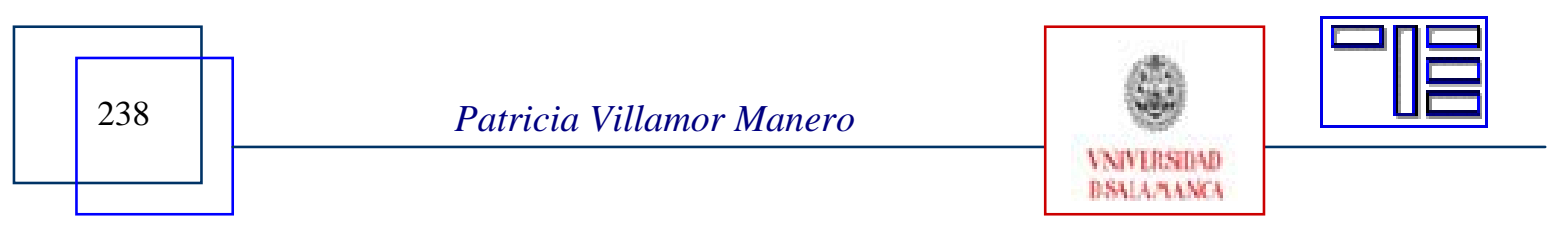


estatutarias (Mitchell, 2001), escuelas con estatuto propio (Whitty, Power y Halpin, 1999). Además de poder especializarse en artes, ciencias, estudios internacionales o en estudiantes con riesgo de absentismo, también presentan una organización particular y adaptada a sus propios contenidos curriculares. La primera escuela charter fue creada en Estados Unidos en 1991 y desde allí se extendieron a otros lugares como Canadá (aunque hasta la fecha sólo Alberta permite su existencia). Pertenecen al sector público (son escuelas subvencionadas por las autoridades públicas en función del número de alumnos matriculados), son gratuitas aunque sus creadores mantienen el derecho a dirigir el centro y su modelo de gestión intenta recoger las características de las escuelas privadas, evitando determinados controles y regulaciones aplicados al resto de escuelas estatales. Las escuelas alternativas y las especializadas curricularmente son centros donde el equipo docente ha diseñado un proyecto pedagógico centrado en una parte del curriculum o en un área transversal del mismo (medio ambiente, enseñanzas deportivas, idiomas, etc.). Las escuelas alternativas se han dedicado tradicionalmente a atender a una población con necesidades educativas especiales.

- Modelos basados en el uso de bonos o cheques escolares.

El término voucher, traducido habitualmente al castellano por bono o cheque escolar, es un documento que representa una cantidad de dinero que la autoridad pública o una institución privada entrega a las familias en pago de los gastos educativos generados por la escolarización (o por la enseñanza recibida en casa) de cada alumno. Cada familia en el ejercicio de su derecho a elegir, determina dónde se dirigen los fondos públicos, ya que los bonos pueden canjearse en cualquiera de las escuelas inscritas en el programa, a las que les suelen exigir determinados requisitos. Aunque la idea de subvencionar directamente a los padres para afrontar los gastos educativos aparecía ya en los escritos de Thomas Paine y de Adam Smith en el siglo XVIII y en el ensayo "On liberty" de Stuart Mill del siglo XIX, la propuesta original de bonos escolares fue realizada por el Nóbel Milton Friedman en el capítulo sexto de su libro "Capitalism and freedom" aparecido en 1962 y posteriormente en su publicación "Freedom to choose" de 1980. Las diferencias en el diseño de los cheques pueden ser enormes. Hay distintos aspectos a tener en cuenta en estos diseños: la población a la que se dirige el bono (todo el alumnado o un sector desfavorecido); las escuelas que pueden participar en el programa (centros públicos y/o privados); cantidad económica que incluye el bono (puede cubrir la totalidad o una parte de las tasas de enseñanza); regulación de las escuelas participantes (amplitud de la autonomía y exigencias por parte de la administración o la empresa que financia los bonos); financiación de los bonos (pública o privada).

- Modelos basados en la desgravación y reducción de impuestos.

Estos modelos permiten obtener una bonificación en los impuestos por el pago de enseñanzas en escuelas privadas. Se desarrollaron bajo la premisa de que los pagadores de impuestos y los padres deberían tener algún poder para dirigir sus impuestos escolares a su sistema educativo preferido y no pagar doblemente por la educación de sus hijos. En realidad, las personas que pagan impuestos no obtendrían directamente mayor control sobre las decisiones tomadas por las escuelas privadas, a pesar de que el dinero de los impuestos apoyaría sus actividades. Dentro del modelo de elección de centro a través de la reducción de las tasas escolares en las deudas fiscales, existen dos procedimientos muy similares: sus nombres originales son Tuition tax credit y Tuition tax deduction. El primero se refiere a un sistema que proporciona reducciones directas en la

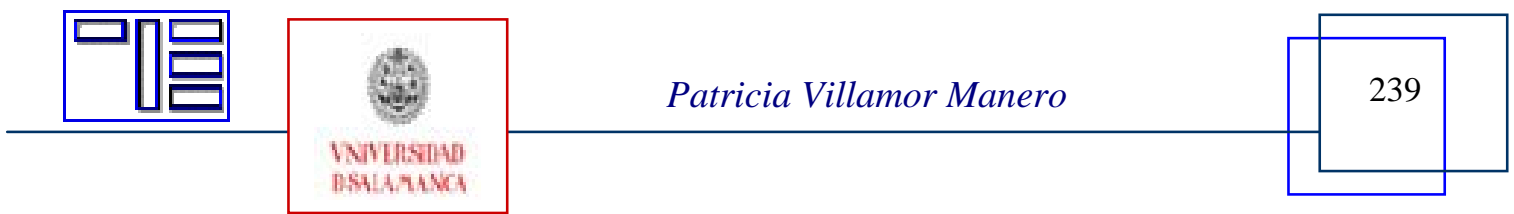


cantidad de retenciones fiscales de un trabajador (en España se referiría a que, una vez calculada la cantidad que debe pagar en concepto de I.R.P.F., Impuesto sobre la Renta de las Personas Físicas, se restan las tasas abonadas a la escuela). El segundo, supone una reducción del nivel de ingresos que declara un trabajador antes de que le sean aplicados los impuestos pertinentes. Algunos autores, como Breen (1984, 23), no encuentran diferencias reales entre los dos procedimientos.

Para Hepburn cualquiera de los incentivos en impuestos es superior, a largo plazo, al resto de opciones, porque incrementan la inversión disponible para educación pero mantienen las decisiones en las manos privadas. De este modo se evita la cuestión de cuánta regulación es necesaria (Hepburn, 1999, 30). Sin embargo, y por el mismo motivo, presenta el problema de beneficiar únicamente a aquéllos que estén utilizando la enseñanza privada. No amplía las opciones para los que sólo contemplan las escuelas públicas o para aquellos cuyos impuestos no permiten descontarse las cantidades totales permitidas.

- Modelos basados en el control de las asignaciones

Existen dos tipos de programas dentro de esta categoría, los que no imponen ningún tipo de restricción en la admisión de alumnos (open enrolment) y los programas de elección universal controlada. Este sistema permite que cada alumno elija cualquier escuela de las participantes en el programa (a menudo fuera del propio distrito escolar) sin que las admisiones sufran ninguna restricción general más allá de las propias de cada centro. Todos los alumnos son distribuidos según sus preferencias, con poca o ninguna consideración de la zona de residencia. En sus comienzos, en los años ochenta en los Estados Unidos, los sistemas open enrolment fueron creados para combatir la segregación en las escuelas pero tuvieron los efectos contrarios, ya que se demostró que una de las causas de la segregación era que los padres blancos utilizaban las políticas de cambios de centro para llevar a sus hijos lejos de escuelas situadas en vecindarios que se convertían en áreas racialmente mixtas y matricularles en escuelas con menor proporción de estudiantes de minorías (Glenn, 1991, 12). La elección universal controlada trata de proporcionar tanta amplitud de elección como sea posible, pero introduciendo algunas restricciones en las decisiones sobre las admisiones. En su origen, pretendían mantener el equilibrio racial de la población general y aunque sería incorrecto decir que la integración se encuentra en la esencia de la elección controlada, constituye el más frecuente instrumento para su consecución. La asignación de los alumnos es realizada por los administradores siguiendo criterios de admisión que pueden variar desde el equilibrio racial o socioeconómico o la zona de influencia, hasta la integración de alumnos con necesidades educativas especiales, en función del objetivo general planteado en cada contexto. Además de definir los criterios que van a regular la entrada de los estudiantes, que en este sistema no pertenecen a cada escuela sino que deben ser aplicados en toda la planificación de las escuelas participantes, normalmente se crea una oficina central desde la que realizan los repartos de estudiantes. Incluso con el mismo criterio prioritario de admisión, existen varias maneras de llevarlos a cabo. Es posible respetar el orden de prioridad manifestado por las familias. También pueden sortearse las plazas entre aquellos que cumplan el requisito prioritario (raza, nivel socioeconómico, etc.), o combinar los dos anteriores.

- Modelos basados en la amplitud del espacio de la elección.

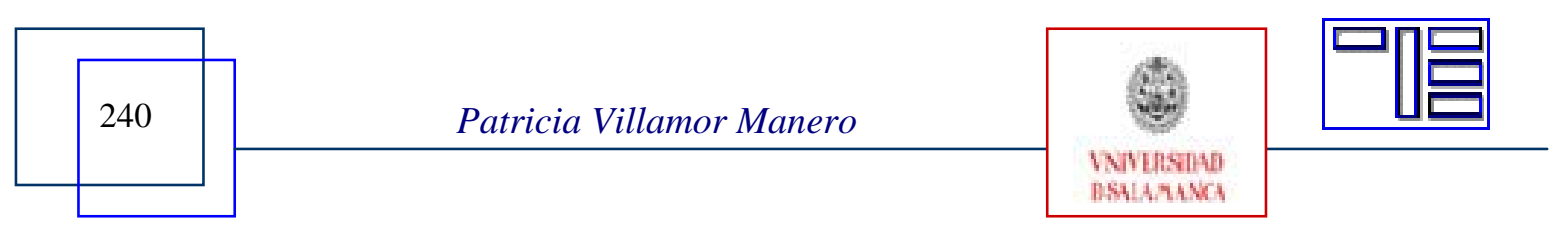


En este tipo de modelos se han incluido los programas de elección intradistrito y los de elección interdistrito. Los primeros están diseñados para permitir a las familias elegir escuela sólo dentro de un determinado distrito o zona geográfica concreta. Normalmente estos planes se establecieron en Estados Unidos junto con políticas de integración, sin aumentar mucho las opciones disponibles para las familias ya que se centraron en la reasignación racial. Se crearon después incentivos para la transferencia entre distritos. Su diseño es el más simple de todos. En España se trataría simplemente de ampliar (como de hecho ha ocurrido en determinadas comunidades) las zonas de admisión de los centros, permitiendo a las familias elegir escuelas alejadas de sus domicilios. Es difícil clasificar todas las formas distintas en que se han llevado a cabo estos tipos de programas. Las más comunes han sido la combinación con modelos de centros con especialización curricular (habitualmente escuelas imán). Por su parte, las experiencias de movilidad interdistritos pretenden permitir a las familias que elijan centros de otras zonas de admisión en las que su domicilio no está originalmente incluido. En general, la aceptación se hace en función del espacio disponible y de no provocar la discriminación racial o socio-económica. En ocasiones, grandes ciudades o estados completos han obligado a todas las zonas a participar, aunque limitando la cantidad de estudiantes que podían realizar la transferencia de escuela y estableciendo mediante una serie de regulaciones, como que ese cambio no afecte a las proporciones raciales de ambos centros o distritos, los emisores y los receptores.

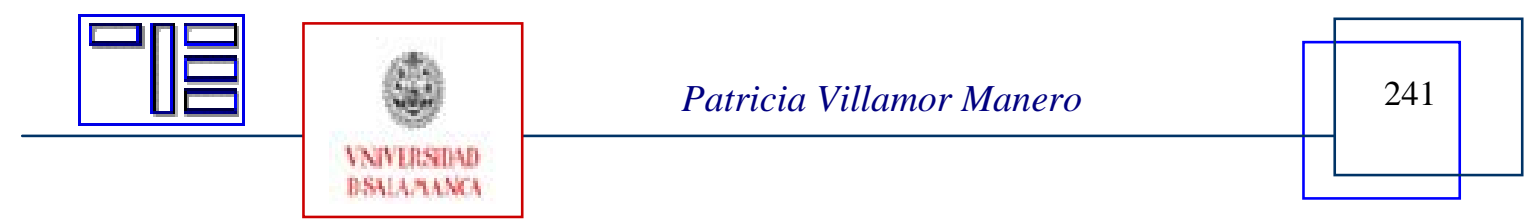




\section{3.- SÍNTESIS DE LOS PROGRAMAS Y EXPERIENCIAS DE ELECCIÓN EN ESTADOS UNIDOS}

\begin{tabular}{|c|c|c|c|}
\hline AÑO & $\begin{array}{l}\text { LUGAR Y } \\
\text { NOMBRE } \\
\text { DEL PROGRAMA }\end{array}$ & $\begin{array}{l}\text { OBJETIVOS Y } \\
\text { DESCRIPCIÓN }\end{array}$ & $\begin{array}{l}\text { TIPO DE } \\
\text { PROGRAMA }\end{array}$ \\
\hline 1968 & $\begin{array}{l}\text { Choctaw County (Ala- } \\
\text { bama) }\end{array}$ & $\begin{array}{l}\text { Proporciona bonos a alumnos que no desean acudir a escuelas locales donde se lleva a cabo el plan } \\
\text { de integración racial. }\end{array}$ & $\begin{array}{l}\text { Bonos escolares con financiación } \\
\text { pública para escuelas privadas no } \\
\text { confesionales. }\end{array}$ \\
\hline $\begin{array}{l}\text { Años } \\
70\end{array}$ & $\begin{array}{l}\text { Eugene } \\
\text { (Oregon) }\end{array}$ & $\begin{array}{l}\text { Extender la libertad de elección de centro de las familias. } \\
\text { Permite que cada familia elija la escuela que más se adapte a sus valores y necesidades. }\end{array}$ & $\begin{array}{l}\text { Elección intradistrito. } \\
\text { Escuelas alternativas. }\end{array}$ \\
\hline 1972 & $\begin{array}{l}\text { Distrito de Lagunitas } \\
\text { (California) }\end{array}$ & $\begin{array}{l}\text { Aumentar la diversidad en la oferta educativa para adaptarse a los valores de los distintos grupos } \\
\text { del distrito. }\end{array}$ & $\begin{array}{l}\text { Escuelas alternativas en el siste- } \\
\text { ma público. }\end{array}$ \\
\hline 1974 & $\begin{array}{l}\text { East Harlem (New York) } \\
\text { Manhattan's Community } \\
\text { School District }\end{array}$ & $\begin{array}{l}\text { Mejorar el rendimiento general del distrito. } \\
\text { El procedimiento de admisión tiene en cuenta las elecciones de los alumnos y los requisitos de } \\
\text { admisión de cada centro. }\end{array}$ & $\begin{array}{l}\text { Elección intradistrito. } \\
\text { Escuelas alternativas. }\end{array}$ \\
\hline 1974 & $\begin{array}{l}\text { Washington } \\
\text { Six School Complex }\end{array}$ & $\begin{array}{l}\text { Prevenir la pérdida de escuelas de la localidad aumentando el número de alumnos residentes. Mejo- } \\
\text { rar la calida y diversidad de los programas ofrecidos. } \\
\text { Se estableció una zona única de admisión y cada centro desarrolló una temática diferente. }\end{array}$ & $\begin{array}{l}\text { Elección intradistrito. } \\
\text { Escuelas temáticas. }\end{array}$ \\
\hline 1976 & $\begin{array}{l}\text { Milwaukee } \\
\text { (Wisconsin) }\end{array}$ & $\begin{array}{l}\text { Lograr escuelas integradas permitiendo elegir la que más se adapte a sus valores y necesidades. } \\
\text { Los criterios de asignación están basados en la elección de los alumnos respetando el equilibrio } \\
\text { racial en las escuelas. }\end{array}$ & $\begin{array}{l}\text { Elección intradistrito. } \\
\text { Escuelas imán. }\end{array}$ \\
\hline 1977 & $\begin{array}{l}\text { Condado de Montgome- } \\
\text { ry (Maryland) }\end{array}$ & $\begin{array}{l}\text { Lograr escuelas integradas. } \\
\text { Aumentar la libertad de elección de centro de la familia. } \\
\text { Los alumnos son asignados a la escuela local, pero se les permite solicitar una diferente si desean. }\end{array}$ & $\begin{array}{l}\text { Escuela imán. } \\
\text { Elección intradistrito. }\end{array}$ \\
\hline 1977 & $\begin{array}{c}\text { Montclair } \\
\text { (New Jersey) }\end{array}$ & $\begin{array}{l}\text { Lograr escuelas integradas. } \\
\text { A partir de } 1985 \text { todas las escuelas se convirtieron en escuelas temáticas y no hay ninguna restric- } \\
\text { ción residencial parra acudir a ellas. }\end{array}$ & $\begin{array}{l}\text { Elección intradistrito. } \\
\text { Escuelas imán. }\end{array}$ \\
\hline $\begin{array}{c}1980- \\
1991\end{array}$ & $\begin{array}{l}\text { Richmond (California) } \\
\text { System for Choice }\end{array}$ & $\begin{array}{l}\text { Ampliar la libertad de elección de las familias mediante el aumento en la diversidad de la oferta. } \\
\text { Lograr escuelas integradas. }\end{array}$ & $\begin{array}{l}\text { Elección intradistrito. } \\
\text { Escuelas temáticas. }\end{array}$ \\
\hline $\begin{array}{l}1980- \\
1988\end{array}$ & St. Louis (Missouri) & $\begin{array}{l}\text { Lograr escuelas integradas, evitando la segregación residencial. Mejorar la calidad de la enseñanza } \\
\text { de los centros del interior de la ciudad. } \\
\text { Los alumnos de zonas residenciales pueden acudir a escuelas del centro de la ciudad y viceversa. }\end{array}$ & $\begin{array}{l}\text { Elección interdistritos. } \\
\text { Escuelas imán. }\end{array}$ \\
\hline
\end{tabular}

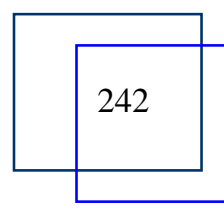

Patricia Villamor Manero
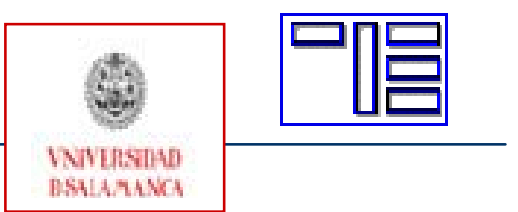


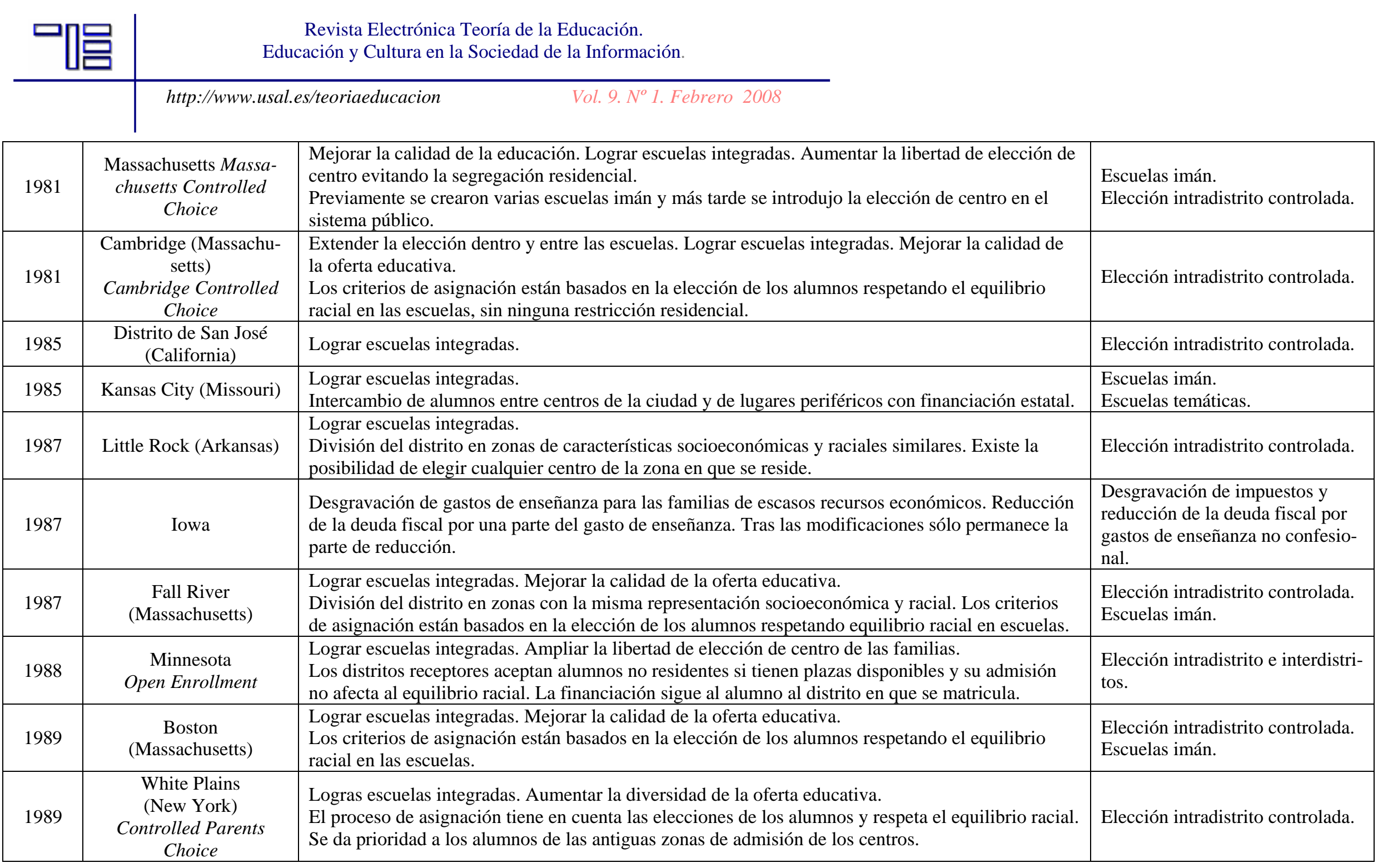

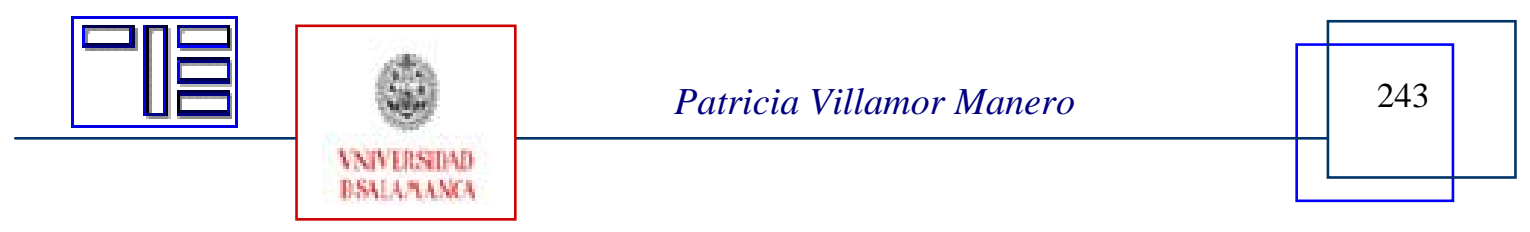




\begin{tabular}{|c|c|c|c|}
\hline 1990 & $\begin{array}{l}\text { Milwaukee } \\
\text { (Wisconsin) } \\
\text { Milwaukee Parental } \\
\text { Choice Program }\end{array}$ & $\begin{array}{l}\text { Extender la libertad de elección de centros aumentando la diversidad de la oferta educativa para los } \\
\text { alumnos de menores recursos económicos. Mejorar la enseñanza recibida por las minorías evitando } \\
\text { su traslado a escuelas fuera del distrito. } \\
\text { Sólo pueden optar a los bonos los alumnos de limitados recursos económicos, que son selecciona- } \\
\text { dos con la aplicación de determinados criterios y un sorteo entre todos los que los cumplan. Pueden } \\
\text { elegirse escuelas privadas entre todas las adscritas al programa. }\end{array}$ & $\begin{array}{l}\text { Bonos escolares con financiación } \\
\text { pública para escuelas privadas. }\end{array}$ \\
\hline 1991 & $\begin{array}{l}\text { Indianápolis (Indiana) } \\
\text { Choice Charitable Trust }\end{array}$ & $\begin{array}{l}\text { Bonos financiados por la Golden Rule Insurance Company dirigidos a alumnos de escasos recursos } \\
\text { económicos para subvencionar una parte del coste de su matrícula en centros privados. }\end{array}$ & $\begin{array}{l}\text { Bonos escolares con financiación } \\
\text { privada para escuelas privadas. }\end{array}$ \\
\hline 1991 & $\begin{array}{l}\text { Massachusetts } \\
\text { Public School Choice }\end{array}$ & $\begin{array}{l}\text { Aumentar la libertad de elección de centro evitando la segregación residencial. } \\
\text { La participación de los distritos es voluntaria en la recepción de los alumnos. La financiación sigue } \\
\text { parcialmente al alumno. }\end{array}$ & Elección interdistritos. \\
\hline 1992 & Minnesota & $\begin{array}{l}\text { Aumentar la diversidad y la calidad en la oferta educativa. } \\
\text { Creación de centros independientes, sin control del distrito, dirigidos por consejos de docentes y } \\
\text { sujetos a menos regulaciones estatales. }\end{array}$ & Escuelas con contrato. \\
\hline 1992 & $\begin{array}{l}\text { San Antonio } \\
\text { (Texas) } \\
\text { Children's Edcuational } \\
\text { Opportunity }\end{array}$ & $\begin{array}{l}\text { Bonos para familias de escasos recursos económicos que subvencionan una parte de los gastos de } \\
\text { la enseñanza en escuelas privadas. }\end{array}$ & $\begin{array}{l}\text { Bonos escolares con financiación } \\
\text { privada para escuelas privadas. }\end{array}$ \\
\hline 1992 & $\begin{array}{l}\text { Milwaukee } \\
\text { (Wisconsin) } \\
\text { Partnership for Educa- } \\
\text { tional Choice }\end{array}$ & $\begin{array}{l}\text { Proporciona a los estudiantes, prioritariamente los de escasos recursos económicos, bonos que } \\
\text { cubren la mitad de los gastos de enseñanza en escuelas privadas. }\end{array}$ & $\begin{array}{l}\text { Bonos escolares con financiación } \\
\text { privada para escuelas privadas. }\end{array}$ \\
\hline 1994 & Michigan & $\begin{array}{l}\text { Mejorar la calidad de la enseñanza y aumentar la diversidad en la oferta. } \\
\text { Creación de nuevas escuelas o transformación de las anteriores en escuelas con contrato. Curricu- } \\
\text { lum centrado en las distintas identificaciones étnicas y filosofías educativas. Admisión de todos los } \\
\text { que la soliciten. }\end{array}$ & Escuelas con contrato. \\
\hline 1995 & $\begin{array}{l}\text { Cleveland } \\
\text { (Ohio) } \\
\text { Ohio Pilot Project } \\
\text { Scholarship Program }\end{array}$ & $\begin{array}{l}\text { Extender la libertad de elección de centro de las familias. } \\
\text { Proporciona bonos para escuelas privadas que cubren una proporción de las tasas de los centros, } \\
\text { cantidad que varía según el nivel de ingresos familiares. Se da preferencia en la obtención del bono } \\
\text { a los alumnos de escasos recursos económicos y a los anteriores participantes. }\end{array}$ & $\begin{array}{l}\text { Bonos escolares con financiación } \\
\text { pública para escuelas privadas. }\end{array}$ \\
\hline
\end{tabular}
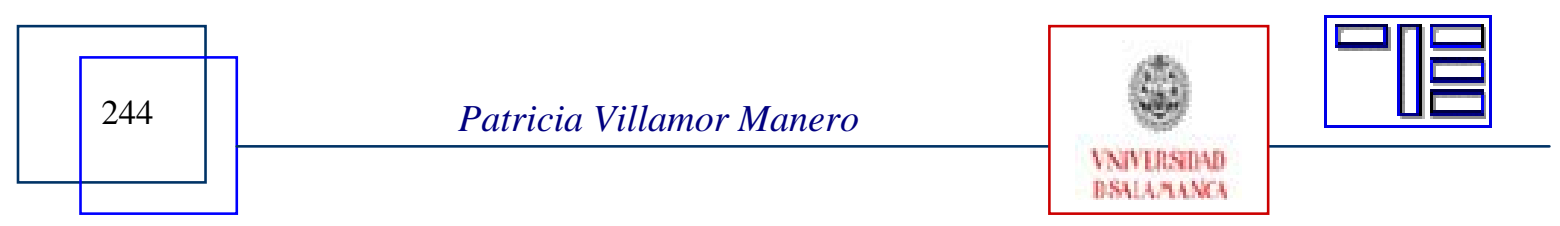


\begin{tabular}{|c|c|c|c|}
\hline \multirow[t]{2}{*}{$\sqcup \square$} & \multicolumn{2}{|r|}{$\begin{array}{l}\text { Revista Electrónica Teoría de la Educación. } \\
\text { Educación y Cultura en la Sociedad de la Información. }\end{array}$} & \multirow[b]{3}{*}{ Escuelas con contrato. } \\
\hline & \multicolumn{2}{|c|}{ http://www.usal.es/teoriaeducacion } & \\
\hline 1995 & Texas & $\begin{array}{l}\text { Extender la libertad de elección de centros aumentando la diversidad de la oferta educativa. Mejo- } \\
\text { rar la calidad de la enseñanza. Motivar la innovación en la educación. } \\
\text { Creación de escuelas independientes con financiación pública. Contrato entre patrocinadores y } \\
\text { organizadores (instituciones públicas o privadas). Exentas de algunas normas estatales. }\end{array}$ & \\
\hline 1996 & $\begin{array}{l}\text { Rockford (Illinois) } \\
\text { Controlled Choice Stu- } \\
\text { dent Assignment Plan }\end{array}$ & Lograr escuelas integradas respetando la elección de centro de los estudiantes. & Elección intradistrito controlada. \\
\hline 1996 & North Carolina & $\begin{array}{l}\text { Extender la libertad de elección de centros aumentando la diversidad de la oferta educativa. Mejo- } \\
\text { rar la calidad de la enseñanza. Motivar la innovación en la educación. } \\
\text { Creación de escuelas independientes con financiación pública o transformación de las ya existentes. } \\
\text { Contrato entre patrocinadores y organizadores (instituciones públicas o privadas). Exentas de algu- } \\
\text { nas normas estatales. Las admisiones no tienen restricciones residenciales y deben respetar los } \\
\text { planes de integración. }\end{array}$ & $\begin{array}{l}\text { Elección intradistrito. } \\
\text { Escuelas con contrato. }\end{array}$ \\
\hline 1996 & $\begin{array}{l}\text { San Antonio } \\
\text { (Texas) }\end{array}$ & $\begin{array}{l}\text { Mejorar la calidad de la enseñanza de los alumnos que acuden a escuelas locales de mala calidad. } \\
\text { Los alumnos matriculados en centros superpoblados y de bajo rendimiento reciben bonos para } \\
\text { subvencionar la enseñanza en escuelas privadas no confesionales. }\end{array}$ & $\begin{array}{l}\text { Bonos escolares con financiación } \\
\text { pública para escuelas privadas no } \\
\text { confesionales. }\end{array}$ \\
\hline $\begin{array}{c}1958 \text { y } \\
1997\end{array}$ & Minnesota & Reducción de la deuda fiscal del 1\% de los gastos de enseñanza en escuelas públicas y privadas. & $\begin{array}{l}\text { Reducción de la deuda fiscal por } \\
\text { gastos generales de enseñanza. } \\
\text { Desgravación de gastos generales } \\
\text { de enseñanza. }\end{array}$ \\
\hline 1997 & Arizona & $\begin{array}{l}\text { Ampliar la libertad de elección de las familias mediante la subvención de las tasas de centros pri- } \\
\text { vados. } \\
\text { Reducción de la deuda fiscal por las donaciones realizadas a organizaciones que proporcionan } \\
\text { becas para acudir a escuelas privadas. }\end{array}$ & $\begin{array}{l}\text { Reducción de la deuda fiscal por } \\
\text { donaciones. }\end{array}$ \\
\hline 1997 & $\begin{array}{c}\text { Albany } \\
\text { (New York) }\end{array}$ & \begin{tabular}{|l|} 
Mejorar el rendimiento de los alumnos de una escuela determinada. \\
Bonos para que alumnos de escuela local se matriculen en centros privados de mayor calidad.
\end{tabular} & $\begin{array}{l}\text { Bonos escolares con financiación } \\
\text { privada para escuelas privadas. }\end{array}$ \\
\hline 1998 & $\begin{array}{l}\text { San Antonio } \\
\text { (Texas) } \\
\text { Edgewood horizon Pro- } \\
\text { gram }\end{array}$ & $\begin{array}{l}\text { Extender la libertad de elección de centros aumentando la diversidad de la oferta educativa a las } \\
\text { escuelas privadas. }\end{array}$ & $\begin{array}{l}\text { Bonos escolares con financiación } \\
\text { privada para escuelas privadas. }\end{array}$ \\
\hline 1999 & $\begin{array}{l}\text { Florida Opportunity } \\
\quad \text { Scholarship }\end{array}$ & $\begin{array}{l}\text { Mejorar la enseñanza recibida por los alumnos matriculados en las escuelas sin rendimiento satis- } \\
\text { factorio. } \\
\text { Subvenciona el traslado de alumnos a centros públicos o privados de más calidad. }\end{array}$ & $\begin{array}{l}\text { Bonos escolares con financiación } \\
\text { pública para escuelas públicas o } \\
\text { privadas. }\end{array}$ \\
\hline
\end{tabular}

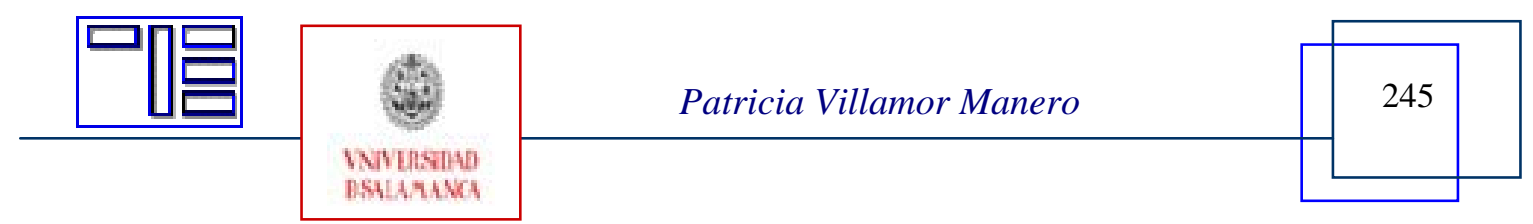


Revista Electrónica Teoría de la Educación.

Educación y Cultura en la Sociedad de la Información.

http://www.usal.es/teoriaeducacion

Vol. 9. No 1. Febrero 2008

\begin{tabular}{|c|c|l|l|}
\hline 1999 & Illinois & $\begin{array}{l}\text { Reducción de la deuda fiscal de las familias de un porcentaje de sus gastos de enseñanza en escue- } \\
\text { las públicas o privadas. }\end{array}$ & $\begin{array}{l}\text { Reducción de la deuda fiscal por } \\
\text { gastos generales de enseñanza. }\end{array}$ \\
\hline 2001 & Pennsylvania & $\begin{array}{l}\text { Las donaciones económicas de las empresas a organizaciones sin ánimo de lucro que proporcionen } \\
\text { becas de enseñanza o a empresas que invierten en el desarrollo de programas de innovación en } \\
\text { escuelas públicas, pueden ayudarles a reducir su deuda fiscal. }\end{array}$ & $\begin{array}{l}\text { Reducción de la deuda fiscal } \\
\text { empresarial por donaciones. }\end{array}$ \\
\hline 2002 & $\begin{array}{c}\text { Estados Unidos (Pro- } \\
\text { grama Federal) } \\
\text { No child left behind }\end{array}$ & $\begin{array}{l}\text { Elevar rendimiento de los alumnos de escuelas locales con evaluaciones de progreso negativas. } \\
\text { Facilita el cambio de centros a los alumnos matriculados en las peores escuelas y proporciona ayu- } \\
\text { da económica extra en los casos más graves. }\end{array}$ & $\begin{array}{l}\text { Elección intradistrito de centros } \\
\text { públicos. }\end{array}$ \\
\hline $\begin{array}{c}\text { Colorado } \\
\text { Opportunity Contract }\end{array}$ & $\begin{array}{l}\text { Favorecer la asistencia a centros privados de alumnos en desventaja socioeconómica, con déficit de } \\
\text { aprendizaje que estén matriculados en escuelas o distritos de bajo rendimiento. }\end{array}$ & $\begin{array}{l}\text { Bonos escolares con financiación } \\
\text { pública para escuelas privadas. }\end{array}$ \\
\hline 2004 & $\begin{array}{c}\text { Distrito de Columbia } \\
\text { D.C. Choice Incentive } \\
\text { Program }\end{array}$ & Favorecer la asistencia a centros privados de alumnos con escasos recursos económicos. & $\begin{array}{l}\text { Bonos escolares con financiación } \\
\text { pública para escuelas privadas. }\end{array}$ \\
\hline
\end{tabular}

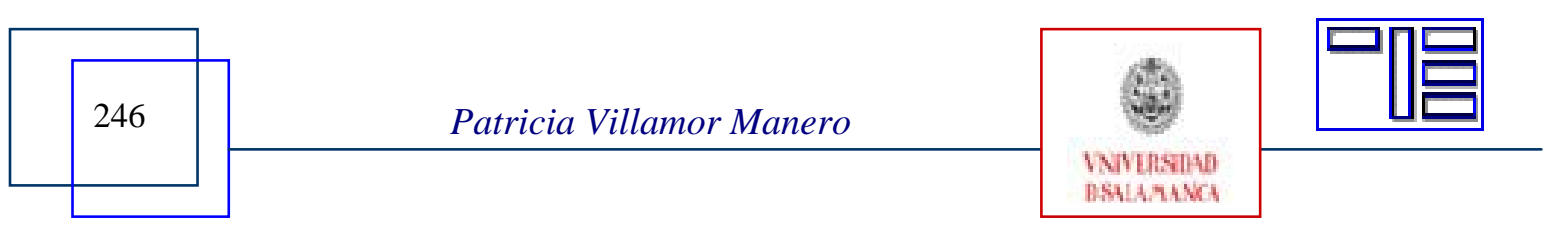




\section{4.- CONCLUSIONES}

La primera conclusión general que se extrae de la información recogida en las tablas anteriores, es que muchos de los objetivos se repiten, incluso con diseños muy distintos. Puede observarse también una evolución en la formulación de estos objetivos a lo largo del tiempo. Durante los años 70 y 80, el objetivo más perseguido fue lograr escuelas integradas racialmente, mientras que en la última década muchos programas han pretendido diversificar la oferta educativa, a menudo incluyendo escuelas privadas en esta oferta.

Además de confirmar cómo pueden conseguirse los mismos objetivos con distintos modelos y diseños, también se demuestra cómo los mismos diseños pueden contribuir a objetivos tan distintos como la integración o la segregación en las escuelas. Evidentemente, aunque el esquema del modelo sea el mismo, las especificaciones serán diferentes.

Haciendo un esfuerzo de síntesis aún mayor (con la pérdida de perspectiva que eso supone), podríamos relacionar los modelos de elección intradistrito o interdistritos controlada, con el objetivo de lograr escuelas integradas; los diseños que se basan en la reconversión o creación de nuevos centros más especializados, con el objetivo de diversificar la oferta educativa; los programas de bonos, con la finalidad de extender la elección a las escuelas privadas; y los planes de reducción de la deuda fiscal y desgravación de impuestos, con la de disminuir el gasto en enseñanza, principalmente en las escuelas privadas, posibilitando que más familias elijan esta opción.

En general, en casi todos los programas se ha optado por limitar la libertad de elección en uno u otro sentido, bien dirigiendo la subvención sólo a determinados colectivos (escasos recursos económicos, minorías, etc.), bien regulando las admisiones con criterios tales como una representación porcentual de las minorías en el centro similar a la de la población general de esa localidad o zona geográfica en la que se sitúa la escuela.

$\mathrm{Al}$ estudiar de forma pormenorizada la implementación de los diferentes diseños de los modelos de libertad de elección, podemos concluir que estos programas nunca se desarrollan de manera aislada de otras políticas o proyectos. Si el objetivo es lograr escuelas integradas, hay que evitar los límites de la segregación residencial pero también introducir cuotas de admisión de las minorías en los centros. Si el objetivo es aumentar la diversidad de la oferta, hay que relajar las regulaciones administrativas y burocráticas para que los centros puedan desarrollar un curriculum propio, pero también eliminar la zonificación en la asignación de alumnos para que realmente sean accesibles para todos.

Los programas de elección, a menudo identificados de manera errónea con los programas de bonos, han sido promovidos por concepciones políticas, filosóficas y pedagógicas muy distintas, y sus diferencias se han visto reflejadas en los objetivos concretos que persiguen y en cómo su diseño se adapta al logro de esos objetivos. De hecho, el enfoque de mercado en la educación no tiene por qué identificarse con la libertad de elección, puede que sí con los sistemas de bonos escolares, pero la elección puede ir mucho más allá. La elección funciona en muchos aspectos y tiene muchos factores positivos que la acompañan. No es, sin embargo, la mano invisible del mercado quien le hace funcionar, sino un buen diseño. La clave para el éxito siempre es el diseño del programa y la coherencia con la política que subyace. Los programas pueden diferir en

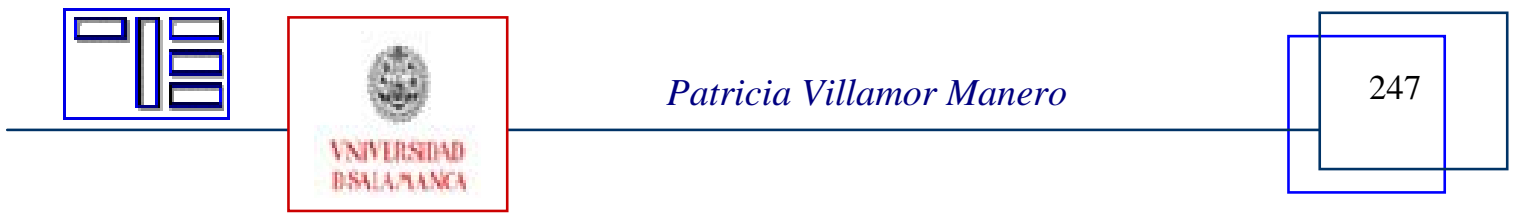


alguno o en muchos de los aspectos de su diseño (amplitud, criterios de admisión, financiación, redistribución, cobertura, etc.) respondiendo a concepciones distintas de calidad y equidad educativa. Los aspectos del diseño que hay que tener en cuenta abarcan desde el modelo concreto bajo el que se desarrolla (bonos escolares, desgravación y deducción de impuestos, control de las asignaciones, amplitud de la elección o una combinación de los anteriores), a su forma de financiación, la regulación y los requisitos de los centros participantes, las medidas complementarias y el procedimiento de elección.

\section{5.- REFERENCIAS}

BENNETT, D.A. (1990): Choice and desegregation. En W.H. CLUNE Y J.F. WITTE, (Eds.), Choice and control in american education. Vol.2: The practice of choice, decentralization and school restructuring. Hampshire, NY, Falmer Press, 125-152.

BREEN, V.D. (1984): Tuition Tax Credits, Journal of the Institute for Socioeconomic Studies, 9 (1), 15-25.

FRIEDMAN, M. (1962): Capitalism and freedom. Chicago, University of Chicago Press.

FRIEDMAN, M. Y FRIEDMAN, R. (1980): Libertad de elegir. Hacia un nuevo liberalismo económico. Barcelona, Grijalbo.

GAURIN, V. (1999): School choice in Chile: Two decades of educational reform. Washington DC, Brookings Institution Press.

GLENN, CH. L. (1991): Why are so afraid of school choice? (And we can learn from Massachusetts), The Public Interest, 102, 2-49.

HENIG, J. R. (1995): Rethinking School Choice: Limits of the Market Metaphor. Princeton, Princeton University Press.

HEPBURN, C.R. (1999): The Case for School Choice. Models from the United States, New Zealand, Denmark, and Sweden. $\quad$ www.fraserinstitute.ca

MITCHELL, S. (2001): Partnerships and charter schools: Contracts in Canadian Reform, Encuentros Sobre Educación, 2, 91-104.

PINI. M.E. Y ANDERSON, G.L. (1999): Política educativa, prácticas y debates en los estados Unidos. Reflexiones sobre América Latina, Revista Iberoamericana de Educación, 20, 137-163.

RAYWID, M.A. (1989): The mounting case for schools choice, en J. NATHAN, (Ed.), Public schools by choice: expanding opportunities for parents, students and teachers. St. Paul, MN, The Institute for Learning and Teaching, 13-40.

VILLAMOR MANERO, P. (2005): La libertad de elección en educación: análisis pedagógico de la situación nacional e internacional. Madrid, UCM.

WHITTY, G.; POWER, S. Y HALPIN, D. (1999): La escuela, el estado y el mercado. Madrid, Morata.

\section{NOTAS}

[ $\left.{ }^{1}\right]$ Friedman (1962) y Friedman (1980)

[2] Villamor Manero, P. (2005): La libertad de elección en educación: análisis pedagógico de la situación nacional e internacional. Madrid, UCM.

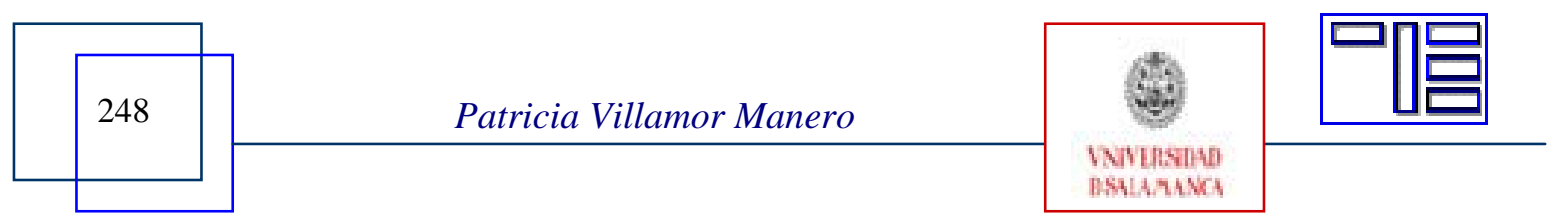

